\title{
Computation of flow and thermal fields in a model CVD reactor
}

\author{
VISHWADEEP SAXENA, K MURALIDHAR and V ESWARAN \\ Department of Mechanical Engineering, Indian Institute of Technology, Kanpur \\ 208 016, India \\ e-mail: kmurli@iitk.ac.in
}

MS received 23 November 2001; revised 5 March 2002

\begin{abstract}
Mixing of coaxial jets within a tube in the presence of blockage has been numerically studied. This configuration is encountered during the modelling of flow and heat transfer in CVD (chemical vapour deposition) reactors. For the conditions prevailing in the reactor, the Reynolds numbers are low and flow can be taken to be laminar and incompressible. The unsteady forms of the governing equations have been solved by a finite volume method that can treat complex three-dimensional geometries. The algorithm is a two-step procedure, wherein the first step predicts the velocity field using an assumed pressure field. The second step corrects the fields using a Poisson equation to obtain the pressure corrections. Advection terms have been treated by a hybrid upwind-central difference technique. The computer code developed is fully three-dimensional, though most computations of the present study have been carried out for two-dimensional geometry. Results have been obtained in the form of velocity vector plots, wall shear stress variation on the block and the tube wall, isotherms and temperature profiles. The flow and heat transfer characteristics of jet mixing have been explored in terms of the Reynolds number, the jet velocity ratio, the axial position of the block, and the blockage ratio. The results obtained show that a proper combination of the process parameters can lead to an improved performance of the CVD reactor.
\end{abstract}

Keywords. Fluid flow; heat transfer; application to CVD reactor; numerical solution.

\section{Introduction}

Confined mixing of jet streams is seen in various industrial processes. Examples are jet pumps, ejectors, and combustion chambers of jet engines. The application of interest in the present study is chemical vapour deposition (CVD). A CVD reactor is generally tubular, with an axially located jet, along with several peripheral jets. The jets carry chemically reacting species, the products of which deposit on the available solid surfaces. In practice, the deposition is

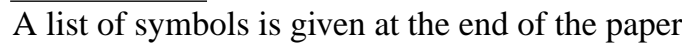


required to take place on a block placed transversely to the jets (Campbell 1996). Substrates that can be grown by this method include silicon hydride and zinc sulphide, materials of importance in electronic devices and infrared sensors. The carrier gas in many applications is argon. When grown at appropriate rates, the deposited material has a polycrystalline form. The CVD reactor can be operated either at a high or a low gas pressure. In the former, the flow field is dominated by buoyancy-driven convection cells arising from density changes within the reactor. When the gas pressure is low, the equivalent Reynolds number is quite small, the flow is laminar and practically incompressible. The flow patterns then contain zones of recirculation that determine the deposition rates of the products of reaction at the solid surfaces. In both contexts, the process is controlled by the details of the flow field (Mahajan 1988, 1996).

The present study is restricted to low pressure CVD reactors. For a typical reactor, the following representative parameters can be assumed (Wadhawan V K 2001, private communication): diameter $=0.1 \mathrm{~m}$, bulk pressure $=30 \mathrm{mbar}$, bulk temperature $=800 \mathrm{~K}$, characteristic flow rate of the inner jet $=500 \mathrm{~cm}^{3} / \mathrm{min}$, the carrier gas being argon. Reynolds number under these conditions is 19 and the Prandtl number 0.7. It can also be shown that buoyancy effects and viscous dissipation are negligible. Thus, laminar incompressible flow conditions can be assumed in the analysis of the flow and thermal fields. Thicknesses of the material deposited in the reactor are of the order of $1-2 \mathrm{~mm}$. Hence the change in the shape and size of the flow space can be neglected during simulation.

CVD reactors presently is use require several months of continuous operation before achieving meaningful levels of the thickness of the deposited material. The deposition efficiency, namely the mass of the material deposited with respect to the mass of the reactants entering the reactor is also exceedingly low, being of the order $0.1-0.2 \%$. There is clearly a definite need to employ appropriate flow rates, velocity ratios and bulk pressures that are correlated with the reactor geometry, so that the process efficiency is improved. The present work has been carried out with this objective.

Recent literature on jet mixing is concerned with primarily turbulent flows, as encountered in applications such as gas turbine combustors. Laminar flow simulations have been presented in earlier studies from a theoretical viewpoint. Ghia et al (1969) reported axisymmetric boundary-layer calculations for laminar mixing of co-axial confined laminar jets, including a non-zero pressure gardient. The mixing was heterogeneous in the sense that the jets had different mean velocities and species concentration. Seider \& Churchill (1971) studied the two dimensional mixing of laminar, incompressible jets with and without chemical reactions using the stream function-vorticity formulation. Shavit \& Laven (1972) investigated the problem of a heterogeneous coaxial jet mixing in the confined tube. The authors concluded that fully developed velocity profiles at the inflow plane and a low density ratio of the mixing streams enhance the formation of recirculation patterns. The flow field in a CVD reactor arises from the heterogeneous mixing of a jet positioned along the axis with others located off-axis at definite locations. The field is further complicated by the presence of blockage. Duverneuil \& Couderc (1992) and Moffat (1992) have reported numerical results for the flow field in a realistic CVD geometry in two dimensions. In a recent study, Park \& Pak (2000) have analyzed flow, heat and mass transfer in a CVD reactor whose configuration is different from that considered in the present work.

In the present study, internal jet mixing in a tubular CVD reactor has been considered. The flow is obstructed by a block placed transversely to the tube axis. The parameters that determine the flow field are the velocity ratio of the jets, the block position and size, and the Reynolds number. Results have been presented in terms of streamlines, velocity vectors and 
the wall shear stress distribution. In order to understand the fluid mixing further, a thermal field calculation in which the incoming jets are at different temperatures is reported. Mass transfer calculations including chemical reactions, as well as the influence of the shape of the block will be carried in a future phase of the study.

\section{Mathematical formulation}

Equations governing laminar incompressible flow are those based on mass balance and the momentum principle which are respectively the continuity and the Navier-Stokes equations (Patankar 1980; Majumdar et al 1992). They are supplemented by the thermal energy equation derived from the conservation of energy prinicple. The governing equations employed in the present work are expressed in cartesian coordinates, and are valid for steady and unsteady flows. The equations have been used in dimensionless form. The following approximations have been made in the present study: (a) the fluid properties are constant; (b) buoyancy effects as well as viscous dissipation are negligible.

The governing equations have to be solved subject to suitable initial and boundary conditions. The initial conditions in all the calculations have been taken to be quiescient. The boundary conditions have been applied with reference to the CVD reactor geometry shown in figure 1. At the walls, the no-slip condition is applicable. On the inflow plane, the velocities within the individual jets are prescribed. Since the basic formulation of the present work is three dimensional, the symmetry condition need not be enforced at the axis. Instead, the dependent variables at the axis were determined by averaging the values at the neighbouring cells. The outflow plane has been located sufficiently far away from the block, so that the zero gradient condition in all velocity components is valid. Pressure is to be specified at only one point in the flow domain; it has been chosen to be zero at the axis on the outflow plane. For the thermal problem, the axial jet is assigned a temperature of unity, while that of the peripheral jet is zero. All solid surfaces have been specified to be perfectly insulating.

\section{Numerical solution}

The governing differential equations of flow and heat transfer in cartesian coordinates have been solved using a finite volume formulation (Eswaran \& Satya Prakash 1998). Here, the governing equations are integrated over an arbitrary prism-shaped cell. The advantage of this

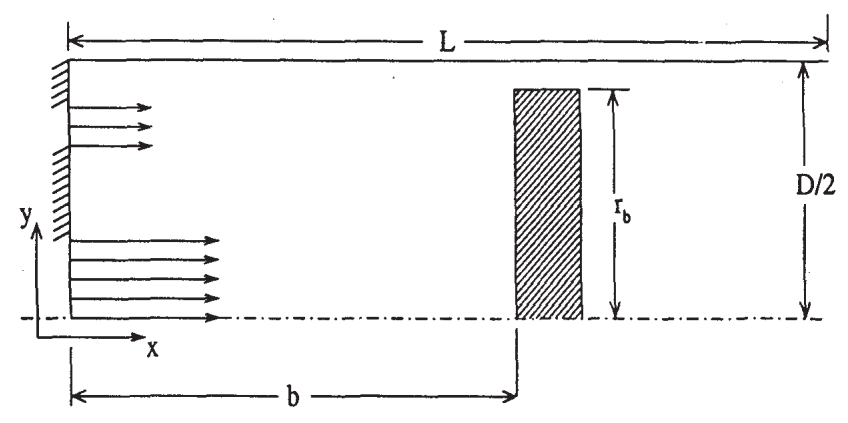

Figure 1. Schematic diagram of the confined jet mixing problem, for an axial to peripheral jet velocity ratio of $V_{r}$. 
approach is that with an appropriate grid generation algorithm, flow in complex geometries can be simulated. Specifically, there is no need for a coordinate transformation. Hence, flow in the tubular reactor can be computed without transforming the equations to a cylindrical coordinate system. A collocated grid in which all dependent variables are prescribed at identical nodal points is used. The numerical algorithm is applicable in three dimensions, and to internal as well as external flow geometries. Although the details are different, the present approach is similar to the proposals of Demirdzic et al (1993) and Majumdar et al (1992). The advection terms have been discretized by a hybrid upwind-central difference method; in most calculations the weight factor in the hybrid scheme was set to be 0.5 . The system of discretized equations for the pressure correction has been inverted by the Gauss-Siedel method. Time discretization is explicit for the momentum and the energy equations, with the exception of the pressure gradient term which is implicit. The continuity equation is also treated by the implicit method. In this respect, the overall scheme is semi-explicit. The time step required for marching is selected on the basis of the CFL criterion. The complete 3D finite volume formulation for Navier-Stokes equations including the algebraic details has been presented by Senthan $\mathrm{et} \mathrm{al}$ (1998).

The computer code employed in the present study has been extensively validated against published numerical results of Kaushik \& Rubin (1995) and the experimental data of Taylor et al (1982) for a curved duct, under laminar flow conditions. The comparison has been found to be uniformly good in all the test cases. The code has also been compared with the velocity profiles of Seider \& Churchill (1971) in the internal jet mixing problem. The comparison was found to be excellent.

The two dimensional solution was derived from the original three dimensional code by considering only a sector of the tube carrying three cells in the angular direction. Two dimensionality could be enforced by applying the periodicity condition in velocity and temperature at the first and the third cells.

The selection of the grid used in the computation was guided by the fact that the annular gap between the block and the tube of the CVD reactor (figure 1) should carry an adequate number of nodes. In all calculations, this number is in the range 8-10. In a two dimensional calculation, a fine grid comprised of $81 \times 101$ cells. In a three dimensional calculation, a grid of $51 \times 51 \times 17$ cells was the finest that could be considered on the available computing platform. On two grid sizes marginally coarser, marked differences were not seen either in the velocity vectors or the wall shear stresses. Hence the solutions presented below can be regarded to be grid-independent. Additional checks such as mass and energy balances at every cross-section of the reactor showed discrepancies of less than 1 and $2 \%$. All calculations were carried out on a P-III, $600 \mathrm{MHz}$ machine with $512 \mathrm{MB}$ RAM. Typical CPU times required for completing a single run were found to be 10 hours in two dimensions and 6-8 days for three-dimensional calculations.

Results obtained in the present study showed that the flow in the reactor is dominated by a recirculation pattern. Hence, there is a need to resolve not just the boundary layers alone, but also several regions away from the boundaries, the recirculation zone for example. A uniform grid was thus opted for, so that all regions in the reactor could be resolved without any bias.

\section{Results and discussion}

Numerical calculations have been carried out for fluid flow and heat transfer over a range of parameters. The parameters include the Reynolds number (Re) based on the incoming 
velocity of the peripheral jets and the characteristic dimension, namely the tube diameter $(D)$. For nominal operating conditions, the typical value of $\operatorname{Re}$ was found to be 19 (§ 1). Results have been presented in the following sections for Reynolds numbers upto 100. Other dimensionless parameters are the block position $b$, the ratio of the axial to the peripheral jet velocity $\left(V_{r}\right)$, and the block size $r_{b}$. The block thickness has not been neglected in the calculations. In most runs, it was set to be equal to three cell thicknesses. The choice of this thickness was motivated by the convenience in applying no-slip conditions.

The range of dimensionless values employed in the simulation are: $V_{r}=5-10 ; r_{b}=$ $0.40-0.45$; and $b=1-2$. The baseline calculation utilized the lower end of the range, with a Reynolds number of 25 . Most calculations have been carried out in two dimensions. Three dimensional runs were carried out only to check the effect of the swirl component of velocity on the recirculation patterns and the wall shear stress. Calculations revealed that the flow patterns quickly stabilized in a few seconds in dimensional real time. In contrast, the thermal field evolved quite slowly. Hence results have been presented only for the steady flow field, while transients have been considered during heat transfer. It is also to be noted that the field of interest is the region ahead of the block facing the incoming jets; fields beyond the block have not been presented.

The transient durations of the flow field with respect to the scalar requires further comment. Under the incompressible flow approximation, the pressure field is set up rapidly and only minor changes take place in the velocity field, with time. In comparison, the thermal field evolves initially through a front moving forward from the inflow plane. Later transients in which the fluid-wall interactions become important are governed by the thermal diffusivity of the fluid, the walls being specified as insulated surfaces, in the present work. In the context of a CVD reactor, the transients in the transported species are governed by surface reactions, diffusion of species within the substrate and higher order effects. In fact, the Schmidt numbers (equivalent to the Prandtl number) for mass transfer of the transported species are of the order 100 and higher. Hence, the flow transients are minor in the application being considered.

Of special interest is the distribution of shear stresses at the solid surfaces, namely the tube wall and the block. A CVD reactor employs reactants carried by the gas. After reactions, products are formed in the solid phase and deposit over the available substrates. A region of high shear would typically have low deposition rates since the local fluid velocities would be high. Similarly, a variation in shear stresses over a surface is likely to result in a nonuniform distribution of the thickness of the deposited material. Thus, the wall shear stress is an indicator of the performance of the reactor itself.

\subsection{Two-dimensional flow fields}

The flow patterns in the tubular reactor with the baseline parameters are shown in figures 2ac. The streamlines in the figure have been drawn by using a commercial package. The plot is made not by calculating the stream function but by joining local tangents to velocity vectors passing through a pre-defined set of points. The central idea behind displaying the streamlines is to emphasize the appearance of a recirculation zone in the CVD reactor, and the near disappearance of the characteristics of the incoming jets. The velocity vectors corresponding to figures $2 \mathrm{a}-\mathrm{c}$ are shown in figure 3 . The influence of increasing the Reynolds number can be seen in these figures. The major observation that emerges from figures 2 and 3 is that the axial and the peripheral jets located at radial locations of $r=0$ and 0.4 mix over a short distance $(x / D<0.5)$ from the inflow plane. The flow field carries a recirculation pattern adjacent to 

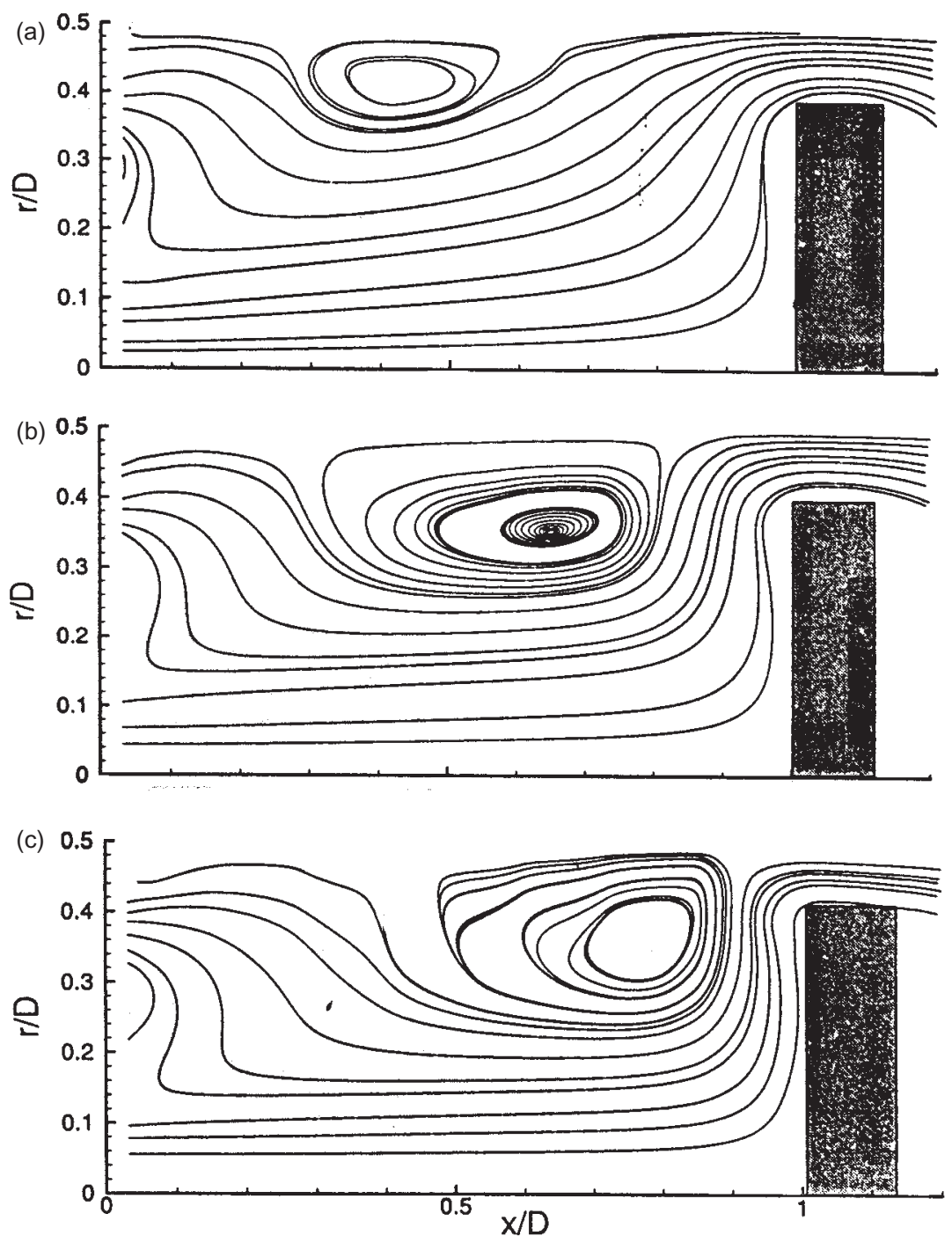

Figure 2. Streamline distribution during confined jet mixing: $V_{r}=5, r_{b}=0.45, \operatorname{Re}=25$ (a), 50 (b) and $100(\mathbf{c})$.

the tube wall. Smaller zones of reversed flow were also seen in the region between the two jets for $x / D<0.1$. With an increase in Reynolds number, the eye of the vortex, namely the centre of the recirculation pattern shifts towards larger values of $x / D$; at the same time the vortex increases in size. This is clearly brought out in figure 3 .

From the viewpoint of the operation of the CVD reactor, the flow fields depicted in figures 2 and 3 indicate early mixing of the constituents of the carrier gas. Using an eye judgement, the distance over which mixing occurs can be estimated to be greater than 0.1 , but definitely less than 0.5 . The deposition on the block takes place when the primary stream moves over it into the annular gap. The particles depositing on the tube walls are those carried by the reversed flow. Since transport between the main stream originating from the jets and the stationary vortex is practically by diffusion, it is to be anticipated that deposition rates at the block 

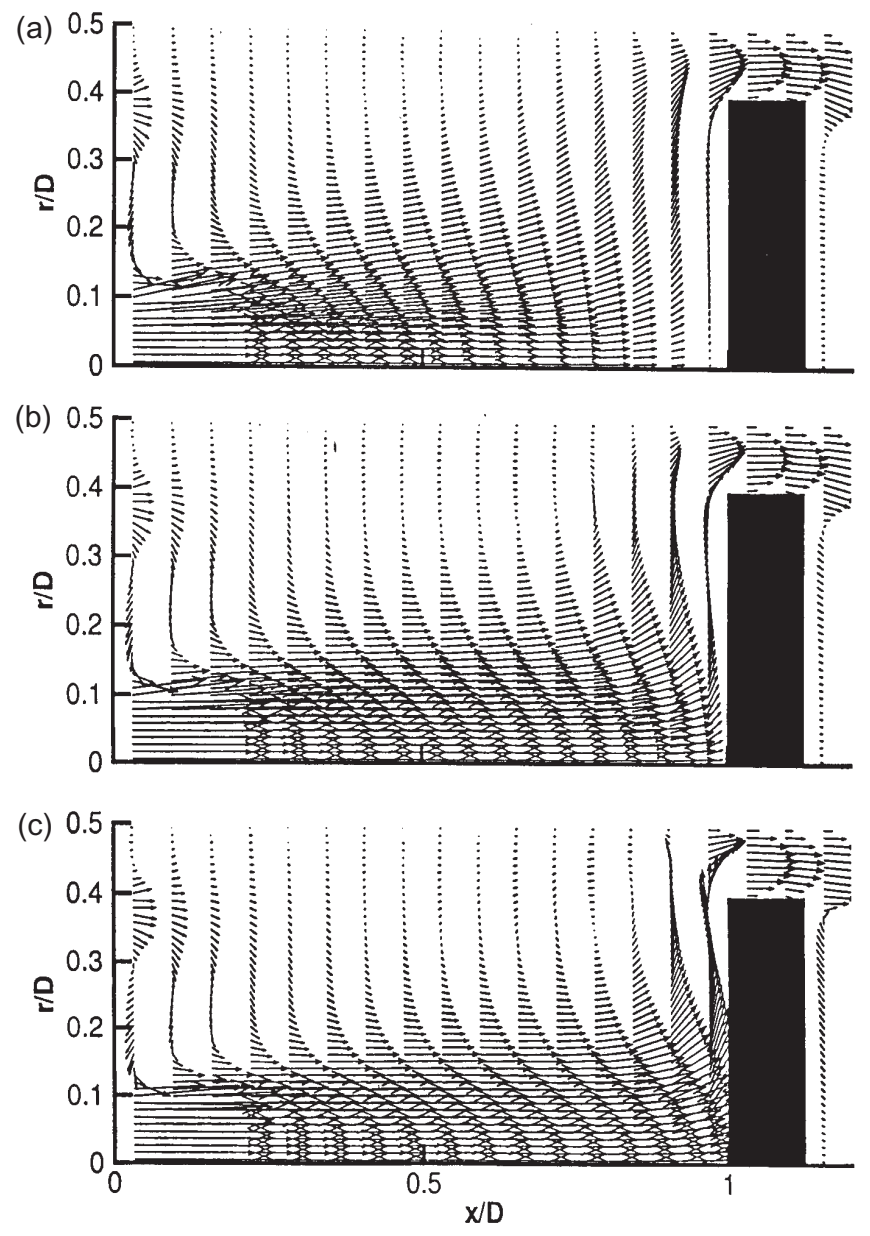

Figure 3. Velocity vector plots in confined jet mixing: $V_{r}=5$, $r_{b}=0.4, \operatorname{Re}=25(\mathbf{a}), 50(\mathbf{b})$ and 100 (c).

would be higher. This should be contrasted against a higher fluid velocity over the block face, leading to a substantial amount of material bypassing the block, and hence the reactor itself. Thus it appears that a proper choice of Reynolds number can optimize deposition rates either on the tube wall or the block.

The streamline plot in figure $4 \mathrm{a}$ shows the effect of increasing the block size to 0.45 . Figure $4 \mathrm{~b}$ is a companion plot when the velocity ratio is increased to 10 . The Reynolds number is 25 in these calculations. The respective velocity vectors are shown in figures $5 \mathrm{a}-$ b. Increased blockage intensifies the zone of reversed flow in terms of circulation, though its axial position is barely affected. Simultaneously, the velocities adjacent to the block are also smaller. The bypassing of the reactants by the main stream is hence reduced, and the transport to the vortex zone, and hence the tube walls is enhanced. The effect of increasing the velocity ratio is to produce a longer recirculation cell, that fills the length of the reactor. Velocities in the region just before the block are higher when compared to a higher blockage ratio. Thus, higher values of both factors $V_{r}$ and $r_{b}$ tend to create flow conditions that will encourage additional deposition at the tube walls, as compared to the block. 

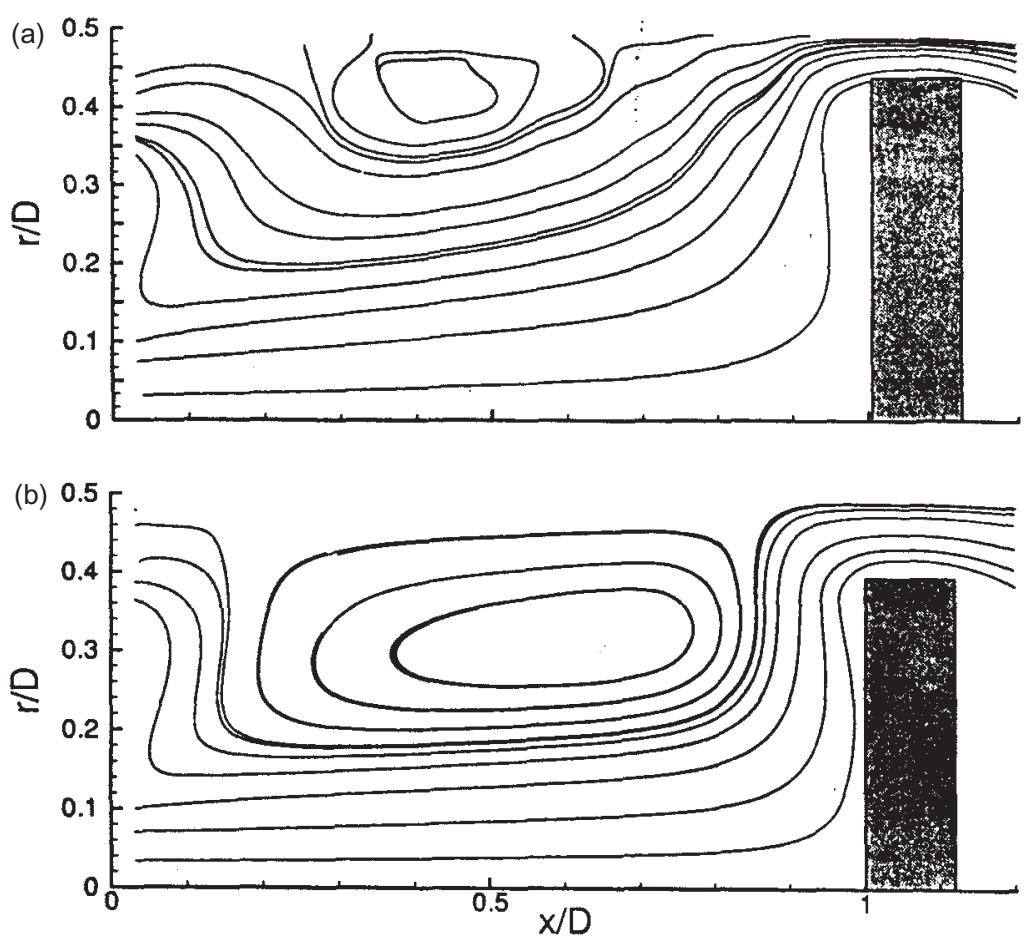

Figure 4. Streamline distribution during confined jet mixing $(\operatorname{Re}=25)$ : (a) $V_{r}=5, r_{b}=0.45$ and (b) $V_{r}=10, r_{b}=0.40$.
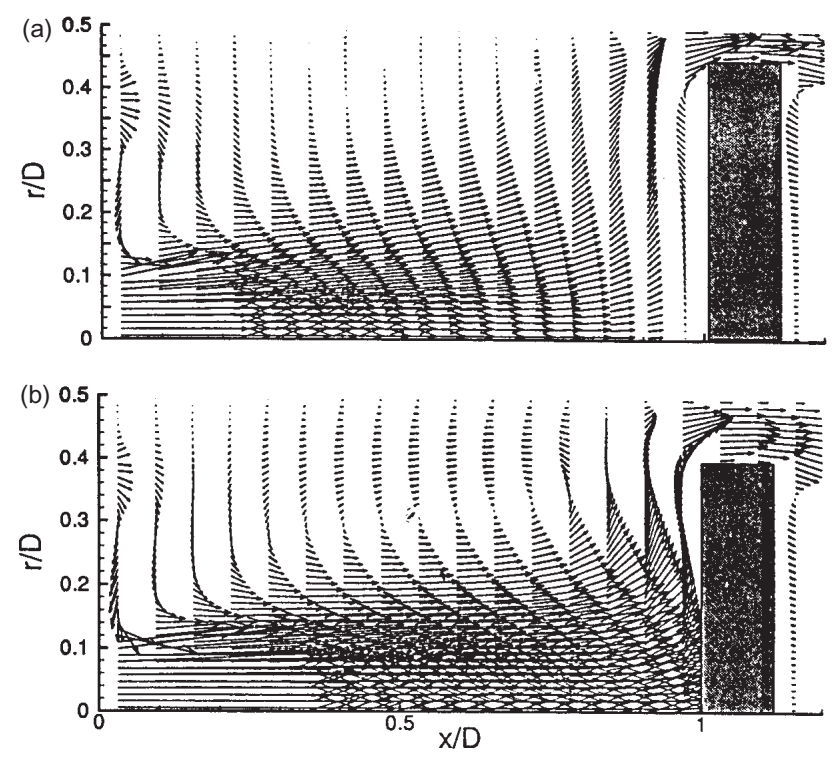

Figure 5. Velocity vector plots in confined jet mixing $(\operatorname{Re}=25)$ : (a) $V_{r}=5, r_{b}=0.45$ and (b) $V_{r}=$ $10, r_{b}=0.40$. 


\subsection{Three-dimensional flow fields}

In the results presented in the previous section, the flow field was taken to be axisymmetric. In this respect, the peripheral jet is taken to be annular (over an angle of $2 \pi$ ) being co-axial with the central jet. In a CVD reactor, the peripheral jets are localised, with 4-6 of them placed circumferentially around the central jet. Thus the flow field is truly threedimensional. To assess the importance of three dimensionality, calculations are carried out with 4 jets placed at angles of $\pi / 2$ at a dimensionless distance of 0.4 from the tube axis. The velocity vectors were then examined on two planes containing pairs of jets and a plane not containing any peripheral jet. Figure 6 shows velocity vectors on the three planes at a Reynolds number of 25. Vector plots on the planes containing the peripheral jets (figures $6 \mathrm{a}$ and b) are practically identical. The difference with figure $6 c$ where the peripheral jet is absent is marginal, except near the inflow plane. The conclusion to emerge from these figures is that the jet flow expands into the tube rapidly, and the jet characteristics are lost beyond an axial location of around $x / D=0.2$. It is also observed that the flow adjacent to the tube wall and the block does not reveal significant three dimensionality. A twodimensional approximation is hence expected to be reasonable. A direct comparison of the
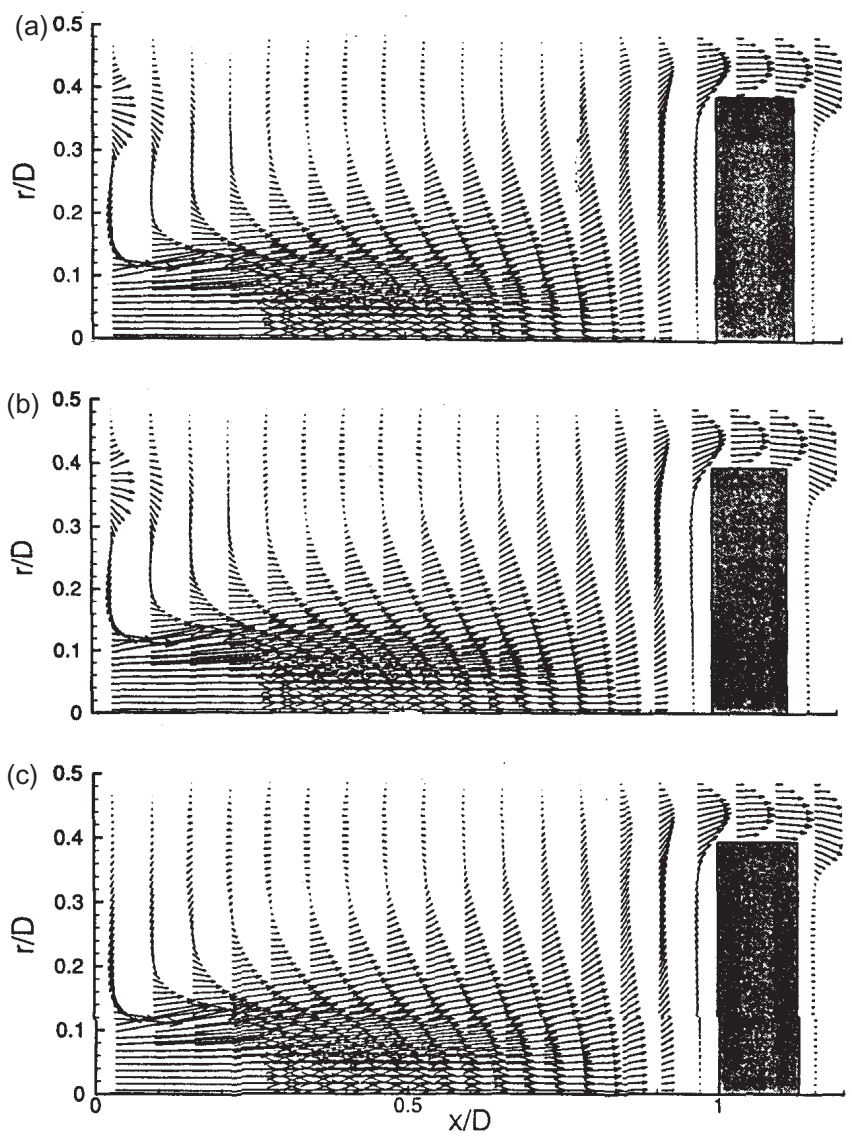

Figure 6. Velocity vector plots, in three-dimensional internal jet mixing $\left(\operatorname{Re}=25, V_{r}=5\right.$, $r_{b}=0.40$ ), in the plane containing (a) the peripheral jets, (b) the second set of peripheral jets and (c) no peripheral jets. 
flow pattern in the reactor with discrete jets with the two-dimensional simulation (figure 3a) can only be qualitative since the volume flow rates in the two geometries are unequal. The match is however favourable, since the size and the position of the vortex can be seen to be quite close.

\subsection{Wall shear stresses}

The shear stress distribution over a solid surface can be correlated with the deposition rates of the products of reaction in a CVD reactor. Zones of high wall shear stress would be sites of low deposition rates. Secondly, non-uniformity in the stress values are indicative of likely variations in the thickness of the material deposited over a period of time in the reactor.

The shear stress distribution over the vertical block has been depicted in figures 7a- $d$ for a variety of parameters. For the baseline configuration (figure $7 \mathrm{a}$ ) the shear stress is a minimum along the central axis $r / D=0$. It increases with the radial coordinate and is a maximum at the outer edge of the block. Bringing the block closer to the inflow plane of the jets ( $b=2$ to $b=$ 1) raises the stress levels, though the distribution tends to become uniform. Increasing the Reynolds number increases the stress values on one hand, and the extent of the non-uniformity on the other. Figure $7 \mathrm{~b}$ shows that Reynolds numbers of 25 and 50 are perhaps acceptable from the viewpoint of uniformity as well as the stress levels. However $\mathrm{Re}=100$ leads to large local shear stresses and can reduce the reactor effectiveness. Figure $7 \mathrm{c}$ shows the effect of increasing the velocity ratio from 5 to 10 . As in the simulation with $R e=100$, a higher velocity ratio
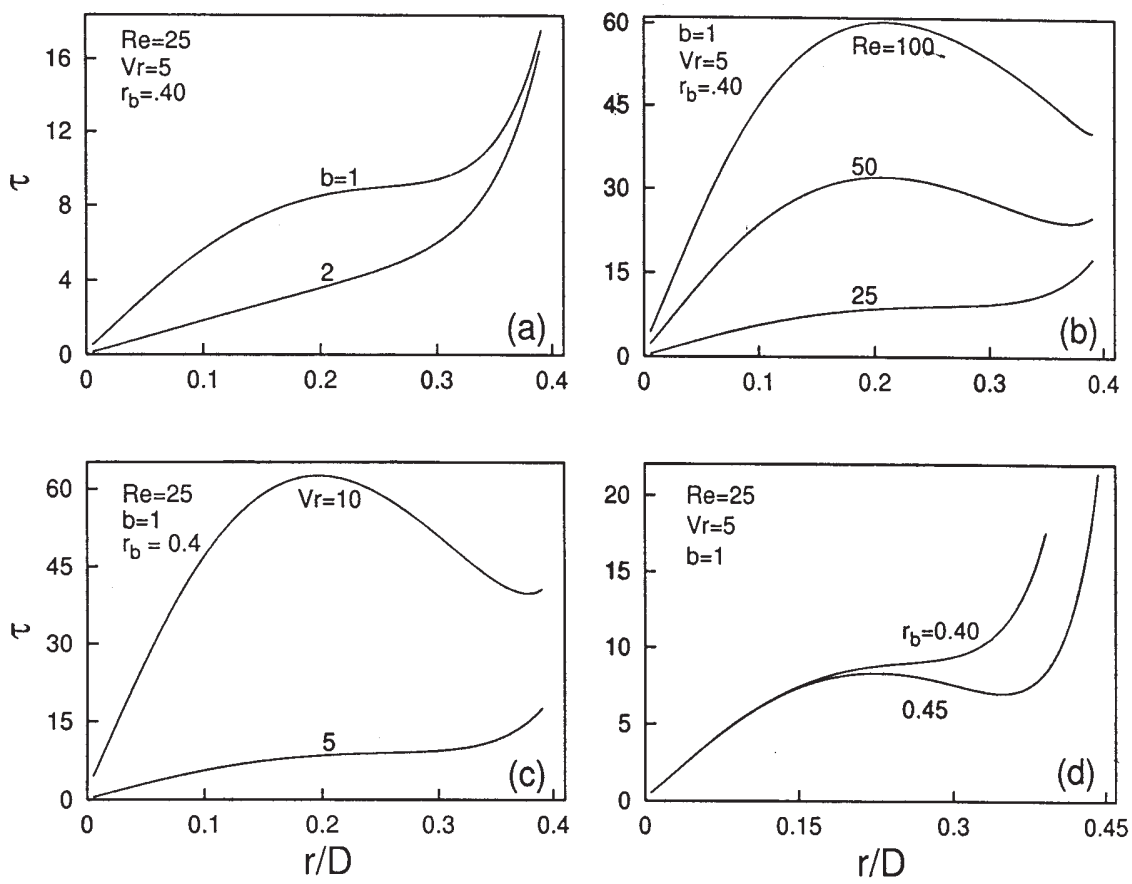

Figure 7. Variation of the wall shear stress on the block in the radial direction: Effect of (a) the block location, (b) Reynolds number, (c) velocity ratio, and (d) block radius. 
produces a sharp peak in the wall shear stress which is not a beneficial effect. A velocity ratio of 5 however produces a reasonably flat profile. Changing the block size from 0.4 to 0.45 leads to a nearly stagnant zone ahead of the block, and a lower stress level over a portion of the surface (figure 7d). Stress levels can also be seen to be quite high at the corners. Increasing the block size however appears to lead to a favourable stress distribution and can be preferred in practice.

The shear stress distribution over the tube wall as a function of the reactor parameters is shown in figures $8 \mathrm{a}-\mathrm{d}$. The shear stress is negative in zones of reversed flow, and becomes positive for larger values of the streamwise coordinate $x / D$. Figures $8 \mathrm{~b}$ and $\mathrm{c}$ show that the stress distribution over the tube surface is only weakly dependent on Reynolds number and the velocity ratio. The block position and size influence the stress levels beyond the separated zone. For $b=2$ the stress values are uniformly small and constant, indicating the potential for considerable deposition of the products of reaction. In reactors where it is desirable to have deposition over the block but not the tube, $b=2$ is however not suitable. To have greater deposition on the block, high shear stresses over the tube walls and low values over the block should be obtained. Figures $8 \mathrm{a}-\mathrm{d}$ show that stresses in the recirculation zone always remain small and result in a build-up of the deposited material on the tube walls.

Figures $9 \mathrm{a}-\mathrm{b}$ show the shear stress profiles along the tube wall during three-dimensional mixing. Reynolds numbers of 25 and 100 have been shown. As discussed in $\S 4.2$, the flow fills the reactor tube and the character of the individual jets is lost. Thus the shear stresses are
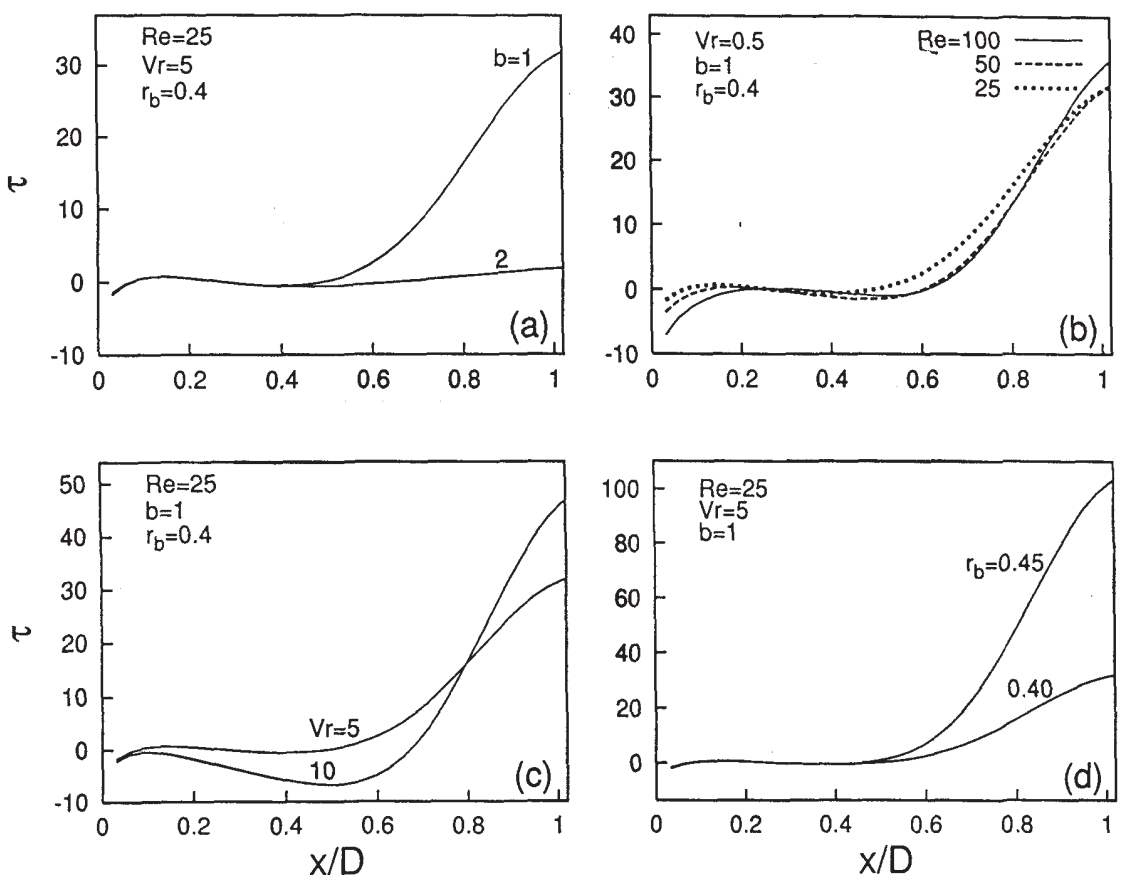

Figure 8. Variation of the wall shear stress on the tube wall in the axial direction: Effect of (a) the block location, (b) Reynolds number, (c) velocity ratio, and (d) block radius. 

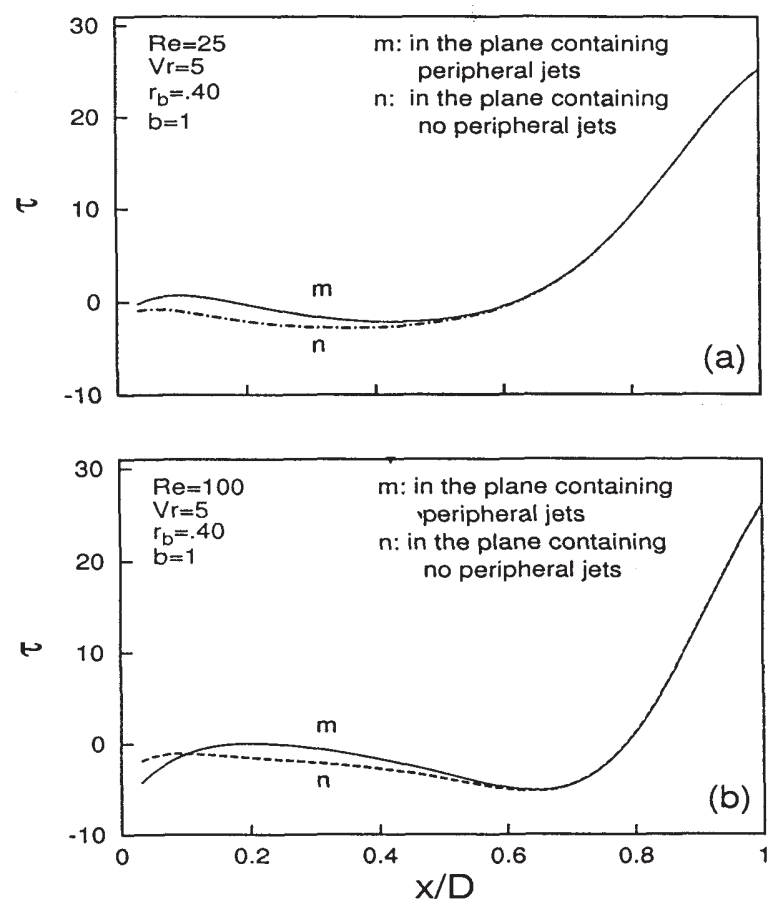

Figure 9. Wall shear stresses during three-dimensional internal jet mixing: $\operatorname{Re}=25(\mathbf{a})$ and $100(\mathbf{b})$.

barely dependent on the azimuthal angle of the reactor. Further, a comparison with figure 8a shows that the dimensionless stress magnitudes in two-and three-dimensional simulation are also quite similar. In this respect, the two dimensional calculations can be taken to be meaningful.

\subsection{Heat transfer}

CVD reactors operate under near-isothermal conditions. The dominant transport phenomenon in the reactor is mass transfer, in the presence of chemical reactions. However, preliminary information about the spread of the species can be derived by solving the transport equation of a single passive scalar. For definiteness, the scalar property has been taken to be temperature, and the transport mechanism as heat transfer. The Prandtl number in all the calculations is 0.7. Schmidt numbers for mass transfer of certain species in the gas phase are close to this value. Hence the trends seen in heat transfer can be expected to carry over to mass transfer. The species concentration of the carrier jets in low pressure CVD reactors is usually quite small, and the mixture can be classified to be dilute. This approximation further reinforces the analogy between heat and mass transfer.

Results have been presented for the time evolution of temperature at distinct points at the block surface and the steady state isotherms in the reactor. Since near-incompressible conditions prevail in the flow field, the fluid dynamic transients last for a very short time. In comparison, those of the scalar field are longer. These expectations were borne out in the numerical simulation as well. The thermal problem considered focusses on the mixing of the hot axial jet with the cold peripheral jet, the walls being thermally insulating. This is equivalent to requiring that the surfaces are chemically passive in the actual mass transfer situation. 

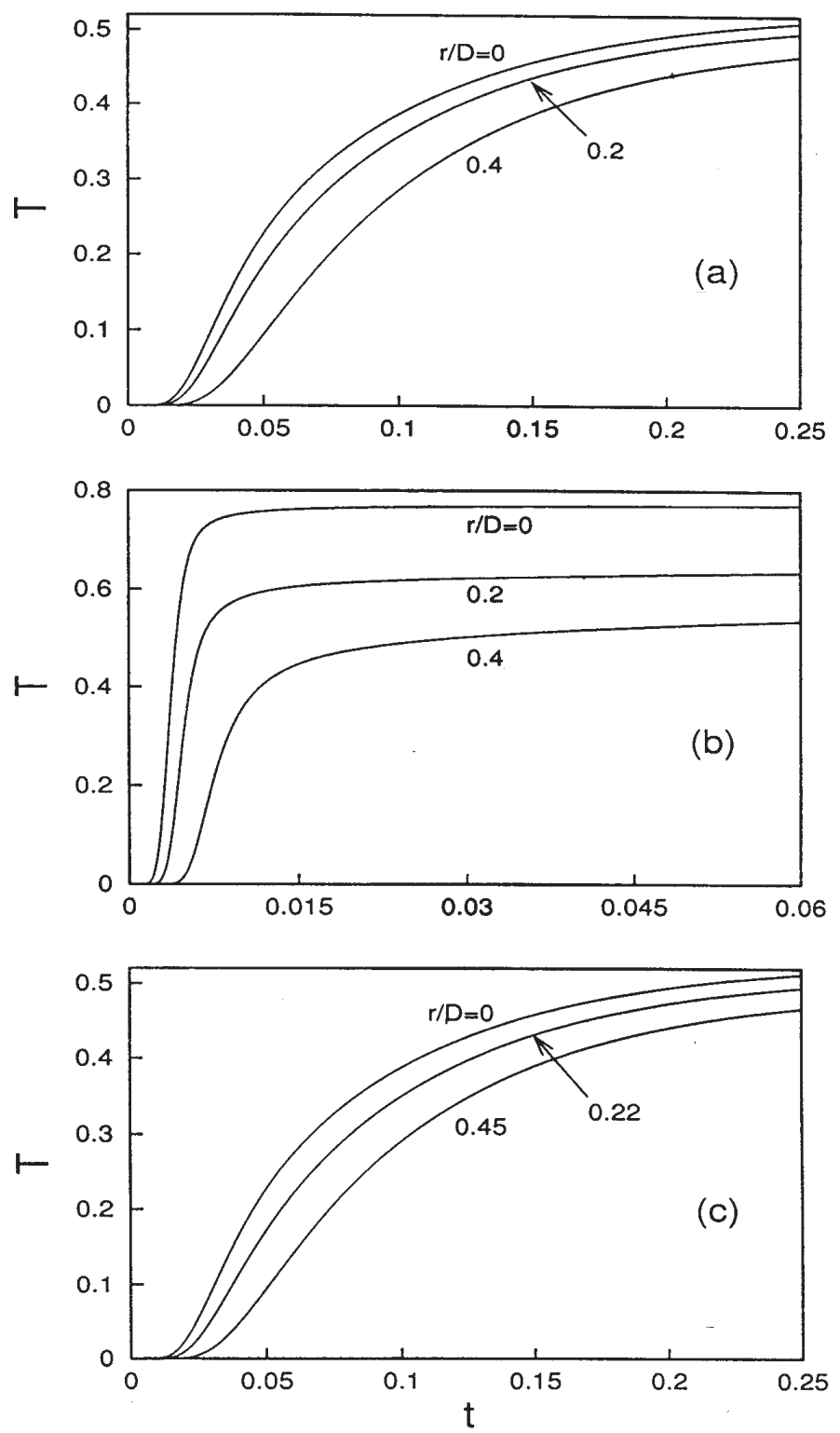

Figure 10. Transient evolution of temperature at points located on the solid block at three radial locations. $b=1, V_{r}=5$; (a) $\operatorname{Re}=25, r_{b}=0.40$; (b) $\operatorname{Re}=100, r_{b}=0.40$; (c) $\operatorname{Re}=25, r_{b}=0.45$.

Figures $10 \mathrm{a}-\mathrm{c}$ show the variation of temperature with time at three radial locations on the vertical block. The highest temperature is recorded at the block centre in every simulation. The temperatures decrease in the radial direction, and a minimum is reached at the corners. The centre of the block is the earliest to attain the steady state, because it is exposed to the axially located jet. At other points, the local fluid velocity is lower and the time scale is affected by diffusion in the gaseous medium. The time required to reach steady state falls rapidly at a higher Reynolds number of 100 (figure 10b). The evolutionary trend also shows 

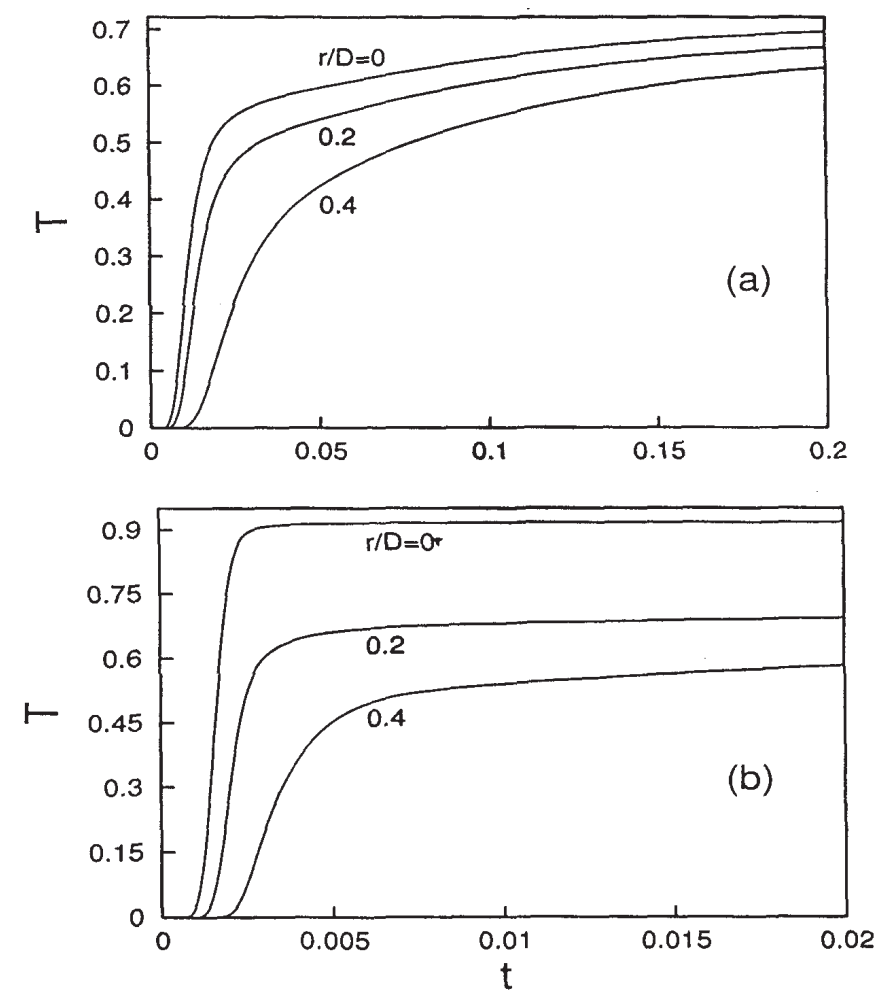

Figure 11. Transient evolution of temperature at points located on the solid block at three radial locations: effect of a higher velocity ratio and Reynolds number. $b=1, V_{r}=10, r_{b}=0.40, \operatorname{Re}=$ 25 (a), and 100 (b).

a sharp increase in temperature with time, typical of advection-dominated problems. The influence of a higher blockage ratio of 0.45 (figure 10c) is however marginal when compared to $r_{b}=0.4$ in figure 10a. In numerical terms, the transients are slightly longer for the higher blockage ratio because of a longer rercirculation zone and a nearly stagnant medium adjacent to the block. In turn, these factors de-emphasize advection effects. The effect of a higher velocity ratio is equivalent to raising the importance of advection effects and lowering the time required to reach steady state. The approach to steady state is front-like (figure 11a), and is clearly brought out with a further increase in Reynolds number (figure 11b).

The time-wise evolution of temperature at selected points over the tube surface showed the trends to be diffusion-like, with a significant delay in reaching steady state. This is to be anticipated because of the presence of the recirculation zone next to the tube wall. The transients were seen to be only weakly affected by changes in the Reynolds number, velocity ratio and the blockage ratio.

Steady state isotherms in the reactor for a variety of operating conditions are shown in figures $12 \mathrm{a}-\mathrm{c}$ and $13 \mathrm{a}-\mathrm{b}$. The isotherms have been labelled, the minimum and maximum temperatures being 0 and 1 respectively. At a lower Reynolds number of 25 , the flow is well-mixed, in the sense that higher temperatures are encountered closer to the tube wall. The degree of mixing decreases at a higher Reynolds number. The temperature gradients along the block length are lower for $\operatorname{Re}=25$, and higher at $\operatorname{Re}=100$. The effect of 


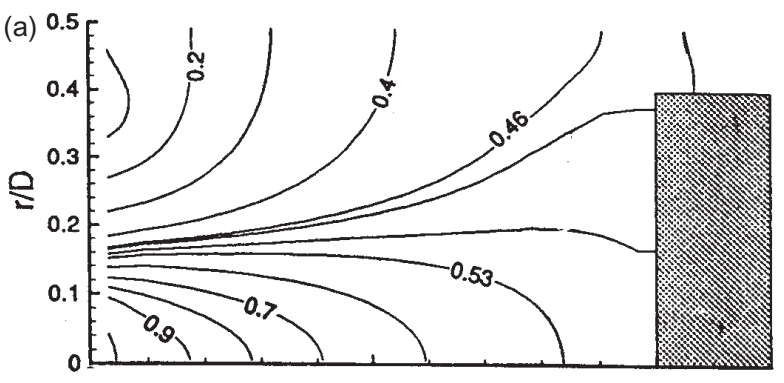

(b)
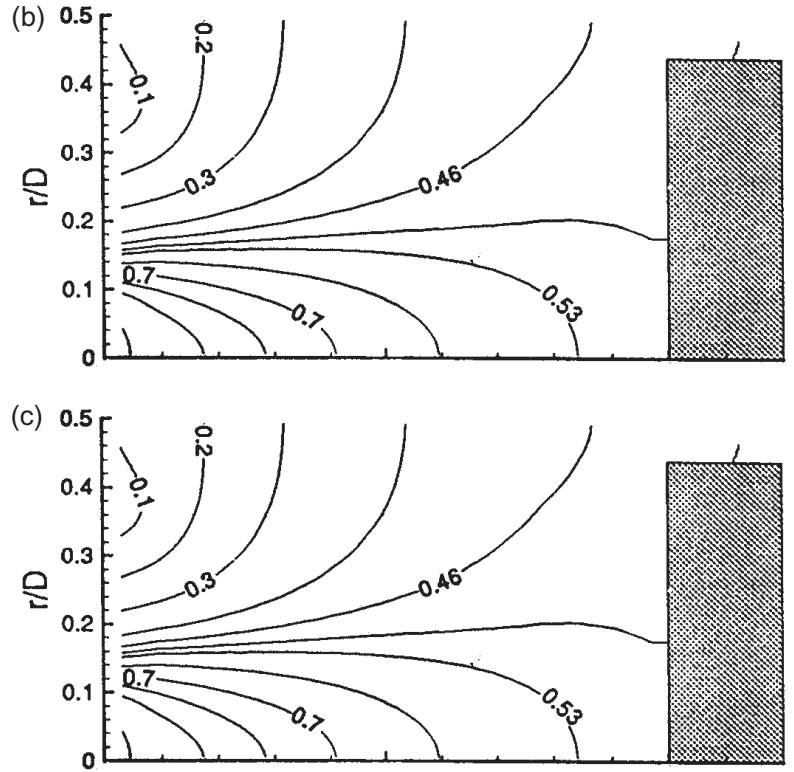

Figure 12. Distribution of isotherms at steady state during confined internal jet mixing $\left(V_{r}=5\right)$ : (a) $\operatorname{Re}=25$, $r_{b}=0.40 ;$ (b) $\operatorname{Re}=25, r_{b}=0.45$ and (c) $\operatorname{Re}=100, r_{b}=0.40$

increasing the velocity ratio is quite similar to increasing the Reynolds number as both cause reduced mixing of the thermal energy of the two streams, and increase the temperature gradients over the block surface. The higher gradients are not useful as they indicate uneven material deposition on the block. Thus $\operatorname{Re}=25$ appears to be the best choice under the assumed conditions. It is to be noted that the shear stress profiles over the block respond in a similar manner to the reactor parameters (figure 6). At the tube wall, the wall shear stress remains small over the recirculation zone (figure 7). The thermal field set up in this region is not constant, and the analogy between shear stress and the steady state temperature does not hold. The temperature distribution shows that material deposition on the tube walls may not be even as one may have expected from viewing the wall shear stress profiles.

\section{Conclusions}

Flow and heat transfer during internal jet mixing in a tubular reactor in the presence of blockage have been numerically investigated. The parameters selected for simulation are 

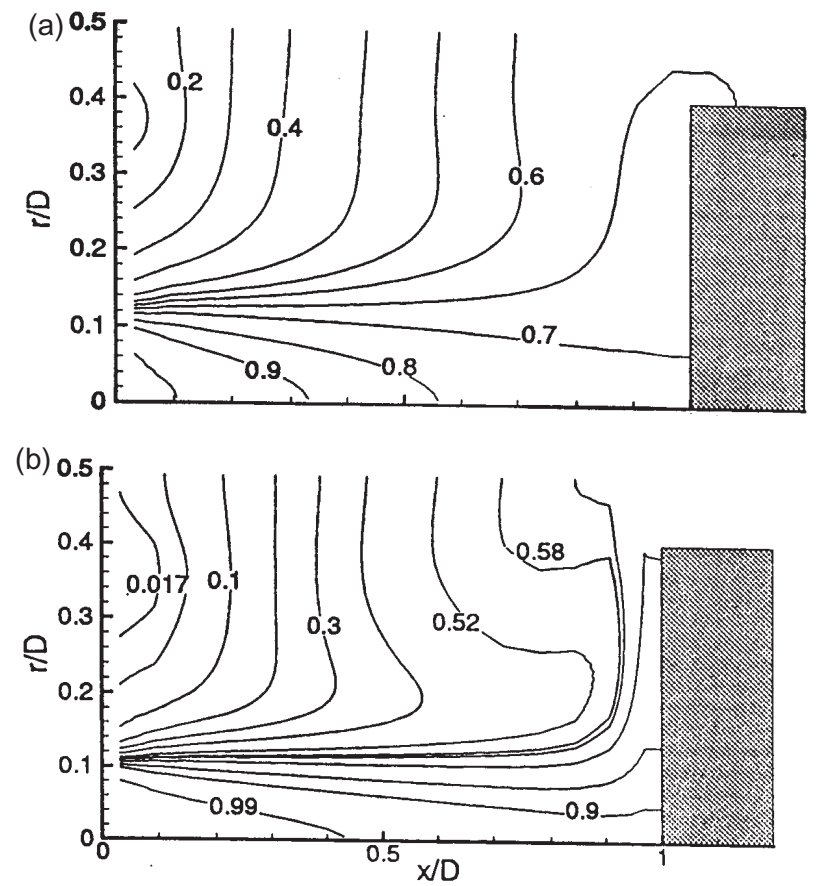

Figure 13. Distribution of isotherms at steady state during confined internal jet mixing $V_{r}=10, r_{b}=0.40$, $\operatorname{Re}=25(\mathbf{a})$, and $100(\mathbf{b})$.

appropriate for low pressure CVD reactors. The major conclusions to emerge from the study are the following.

(1) The incoming jets expand into the tube over a short distance, and the field is close to axisymmetric elsewhere in the reactor. The two-dimensional approximation is hence valid particularly when the focus is on flow patterns adjacent to the block.

(2) The flow field contains a recirculation pattern near the tube wall for all combinations of parameters considered. Consequently, the shear stress levels in this zone are quite small and heat transfer is diffusion dominated. In contrast, the flow field before the block is sensitive to the external parameters such as Reynolds number and velocity ratio. These can be varied in practice to achieve low and reasonably constant shear levels over the block.

(3) The effect of raising the velocity ratio is similar to raising the Reynolds number as far as the shear stress distribution, thermal transients and temperature gradients at the block are concerned. It can cause effects detrimental to the even deposition of material at the block. Increasing the blockage ratio produces nearly stagnant conditions upstream of the block.

\section{List of symbols}

$b \quad$ Dimensionless location of the block;

$D$ tube diameter (m) also characteristic dimension;

$\operatorname{Pr}$ Prandtl number of the carrier gas in the reactor;

$r$ radial coordinate $(\mathrm{m})$;

$r_{b} \quad$ block to tube diameter ratio;

Re Reynolds number based on the peripheral jet velocity and the tube diameter;

$t \quad$ dimensionless time, scaled by $D^{2} / \alpha$; 
$T$ dimensionless temperature scaled by the temperature difference between the hot and the cold jet streams;

$V_{r} \quad$ axial to peripheral jet velocity ratio;

$x \quad$ axial coordinate $(\mathrm{m})$;

$\alpha \quad$ thermal diffusivity of the carrier gas;

$\tau \quad$ dimensionless wall shear stress scaled by $\rho U^{2}$.

\section{References}

Campbell S A 1996 The science and engineering of microelectronic fabrication (Oxford: University Press)

Demirdzic I, Lilek Z, Peric M 1993 A collocated finite-volume method for predicting flow at all speeds. Int. J. Num. Methods Fluids 16: 1029-1050

Duverneuil P, Couderc J-P 1992 Two dimensional modeling of low pressure chemical vapour deposition hot wall tubular reactors. J. Electrochem. Soc. 139: 296-305

Eswaran V, Satya Prakash 1998 A finite volume method for Navier-Stokes equations. Proceedings of the third Asian CFD Conference Bangalore, vol 1

Ghia K M, Todra T P, Laven Z 1969 Laminar mixing of hetrogeneous Axisymmetric Coaxial Confined Jets. AIAA J. 7: 2072-2078

Kaushik S, Rubin S G 1995 Incompressible Navier-Stokes solutions with a new primitive variable solver. Comput. Fluids, 24: 27-40

Mahajan R L 1988 Analysis for control of chemical vapor deposition. Trans. ASME, Journal of Dynamical Systems, Measurement and Control 120: 164-169

Mahajan R L 1996 Transport phenomena in chemical vapor-deposition systems. Advances in Heat Transfer 28: 339-425

Majumdar S, Rodi W, Zhu J 1992 Three-dimensional finite volume method for incompressible flow with complex boundaries. ASME J. Fluids Engg. 114: 496-503

Moffat H K 1992 Numerical modeling of chemical vapour deposition porcesses in horizontal reactors $\mathrm{Ph} \mathrm{D}$ thesis, Dept. of Mechanical Engineering, Univ. of Minnesota

Park K-W, Pak H-Y 2000 Characteristics of three dimensional flow, heat and mass transfer in a chemical vapour deposition reactor. Numer. Heat Transfer A 37: 407-423

Patankar S V 1980 Numerical heat transfer and fluid flow, (New York: McGraw-Hill)

Senthan S, Eswaran V, Biswas G, Muralidhar K, Dhande S G 1998 Numerical simulation of unsteady three-dimensional flow around an elongated body moving in an incompressible fluid. Tech. reports No. 1-4, submitted to Defence Res. Dev. Lab. Hyderabad

Seider W D, Churchill S W 1971 Confined jet mixing in the entrance region of a tubular reactor. AICHE J. 17: 704-712

Shavit G, Laven Z 1972 Analytical and Experimental Investigation of Laminar Mixing of Confined Hetrogeneous Jets. AIAA J. 11: 352-358

Taylor A M K P, Whitelaw J H, Yianneskis M 1982 Curved ducts with strong secondary motion: velocity measurements of developing laminar and turbulent flow. ASME J. Fluids Engg. 104: $350-359$ 